Voix et Images

\title{
Poésie : lorsqu'il est question de répertoire... Perrault, Charron, Des Roches, de Bellefeuille et les Franco-Américains
}

Jean Fisette

Volume 3, numéro 2, décembre 1977

Victor-Lévy Beaulieu

URI : https://id.erudit.org/iderudit/200111ar

DOI : https://doi.org/10.7202/200111ar

Aller au sommaire du numéro

Éditeur(s)

Les Presses de l'Université du Québec

ISSN

0318-9201 (imprimé)

1705-933X (numérique)

Découvrir la revue

Citer cet article

Fisette, J. (1977). Poésie : lorsqu'il est question de répertoire... Perrault,

Charron, Des Roches, de Bellefeuille et les Franco-Américains. Voix et Images,

3(2), 325-328. https://doi.org/10.7202/200111ar d'utilisation que vous pouvez consulter en ligne. 


\section{Poésie: lorsqu'il est question de répertoire... Perrault, Charron, Des Roches, de Bellefeuille et les Franco-Américains}

Dans sa prestigieuse collection "Rétrospectives", l'Hexagone nous propose cette année Gélivures de Pierre Perrault. Premier fait à signaler, c'est que ce recueil ne contient que des textes inédits (ce qui s'appelle entrer par la grande porte, directement dans la tradition récente). Comme quoi la vague de la grande production de l'Hexagone n'est pas encore morte.

La notice blographique, annexée au livre, présente Gélivures comme un «grand poème en six chants" auquel l'auteur aurait travaillé dix ans. Cette présentation pourrait susciter des craintes: on sait que les œuvres poétiques d'envergure ont rarement emporté la palme du succès, et ici, je songe à la Suite marine de R. Choquette, à la Légende d'un peuple de L. Fréchette. Et pourtant, bien qu'il soit encore tôt pour faire une telle affirmation, je ne croirais pas que Gelivures soit promis à une si triste destinée.

Certes l'auteur a du souffle, du vent dans les voiles, et il en faut. Par contre, cette poésie - qui n'est pas narrative - ne cherche pas à s'imposer comme un massif solitaire; chaque poème est précédé d'une épigraphe, vers, strophe ou fragment, emprunté à un poète québécois: Miron, Giguère, P.-M. Lapointe, A. Hébert, J. Brault, R. Lasnier, et j'en passe: la volonté d'enracinement culturel y est évidente.

Mais encore plus, chacun de ces poèmes devient, par une sorte d'effet de mimétisme, prolongement, récriture du texte d'origine. L'épigraphe constitue le seul titre: c'est à dire qu'il devient à la fois, sujet, prétexte, moule. Tel:

\footnotetext{
Adorable femme des neiges ROLAND GIGUĖRE 
en t'aimant neige sur neige je bute à cet excès de sang héréditaire en nuée de guêpes florescentes qui s'échappe de tes écorces et m'assaille à tout rompre

sang froid qui s'enflamme et ne recule devant rien ...(p. 182-183)

Quiconque a quelque peu pratiqué la poésie de l'Hexagone reconnaîtra là une condensation de Giguère ("Adorable femme des neiges") et de l'inoubliable « Janvier - Quêtes de chaleurs" de P.-M. Lapointe (Pour les åmes).

Ainsi les textes de Gélivures se fondent sur une intertextualité (restreinte); voire plus, ils le créent cet inter-texte. Je dirai donc de ce recueil que, loin d'apporter un renouveau d'inspiration ou de formalisation, il consolide une certaine école, la constitue définitivement comme objet privilégié. Compte tenu de la conjoncture politique, nous assistons maintenant à la consécration d'une institution culturelle.

Mais il y a un autre aspect: on retrouve le P. Perrault du Règne du jour et des Voitures d'eau, ainsi que des premiers poèmes égrenant, comme un chapelet, les premiers établissements français le long du fleuve. A savoir la fascination un peu naïve devant l'époque idyllique de la découverte d'une terre vierge, le goût d'un retour aux sources ancestrales. Évidemment c'est le langage - plus précisément le vocabulaire - qui sert d'alibi: n'est-ce pas là le plus important pour le poète? A preuve les quelques épigraphes de Jacques Cartier, du Baron de Lahontan et les titres du recueil, des chants: GÉLIVURES, NEIGERIES, FROIDURETÉ et CORNOUAILLES. Ces mots paraissent comme des fruits juteux dans lesquels on mord: GÉLIVURES, c'est gravures, levure, pure, jure, etc. (plutôt que le stérile mot: gerçures); NEIGERIES, c'est la dureté et la durée des hivers; CORNOUAILLES, ce sont les cornes (omniprésentes dans ce chant), les fiançailles, les semailles, etc.

Je laisse au lecteur, chacun à sa façon, de poursuivre ce jeu d'associations, du déploiement de l'imagination linguistique... C'est qu'il y a ici un évident plaisir des sonorités, de l'éveil, par ce biais, des images.

Par cette voie, P. Perrault jette un pont par-dessus I'histoire, tentant, dans un projet grandiose, de colmater deux cents ans d'histoire, comme s'il ne s'agissait là que d'une brèche. Réussit-il ? Le projet est-il viable? Là n'est pas la question. Plutôt reconnaître que la poésie (surtout moderne) privilégie l'ellipse; et ne pas demander au poète d'être historien!

Pour vulgariser un peu, je dirai qu'il y a une parenté certaine entre cette poésie, à la fois neuve dans sa formalisation et ancestrale dans son inspiration, et, d'autre part, cette mode, de plus en plus répandue, du décapage de vieux meubles en pin et de la restauration des vieilles demeures. 
Les Herbes rouges ont dépassé le cap des cinquante numéros. Peut-être n'a-t-on pas suffisamment examiné cette production qui fait maintenant partie de notre paysage littéraire. Et l'on saura gré aux frères Hébert d'avoir tenu cette gageure. La formule du périodique court consacré à un seul auteur me semble des plus heureuses en ce sens qu'un auteur n'est jamais tenu de produire un texte, un genre définitif: il est toujours appelé à se reprendre. D'autre part cette formule de la récidive a un effet de cohésion sur la collection puisque les auteurs sont appelés à se lire, se commenter mutuellement. Miron parlait, à propos de l'Hexagone, de carrefour (plutôt que d'école); le terme de carrefour me semble encore plus juste dans le cas des Herbes rouges.

Les questions essentielles de la modernité s'y profilent dans une scène quasiment carvanalesque, de la recherche, des tentatives et essais, des confrontations aussi. Les Herbes rouges, c'est à mon avis le lieu essentiel du dynamisme dans notre monde littéraire actuel. Lès noms importants d'aujourd'hui et de demain y figurent certainement.

$\mathrm{J}$ 'en reçois trois numéros. François Charron ( $\mathrm{Du}$ commencement à la fin, $n^{\circ s} 47-48$ ) en rapport aux (en raison des) textes antérieurs se livre à un renouvellement en profondeur de son écriture; sa phrase antérieure qui il faut bien l'admettre - était assez prosaïque, se hachure; comme si le texte, libéré, se mettait soudainement à se formaliser de lui-même; il y a là une matérialité du langage qui prend l'initiative. Lire par exemple:

la multiplicité des courants

marées affluents

la distance nous renvoie la figure d'un

couloir franchi (bloqué) frôlant l'extérieur

à perpétuité les rives le désert la forêt

la reproduction se fait depuis la mère et la

crise interne des matières en flammes

des ensembles tourmentés

$\ldots \ldots \ldots \ldots$ (p. 18)

Peut-être Charron commence-t-il vraiment à écrire.

Roger Des Roches, c'est le classique des Herbes rouges (la Vie de couple nos $50-51$ ). On y retrouve la même virtuosité d'écriture, les mêmes effets de prolifération du signifiant, de démultiplication des effets de sens. Ainsi, ce court fragment:

superbe susperme comme corps vautre: "Belle allurée"

(mais dans l'Histoire de qui cette langue de charmes?):

aurions nombreux peaux licites ou non renversés avec ahs

que la face du monde

On ne peut que souhaiter qu'un jour Des Roches produise un essai de réflexion sur l'écriture moderne: il aura certainement beaucoup à nous apprendre.

Normand de Bellefeuille est, à mon avis, un des auteurs qui promettent le plus: si son écriture est moins formaliste, s'éloignant des mécanis- 
mes de l'automatisme, il n'en demeure pas moins que les questions de l'heure y sont toujours présentes. Ainsi, les derniers textes, les Grandes Familles suivi de Textes pour la jeune sœur $\left(n^{\circ} 52\right)$ sont précédés d'épigraphes d'Hélène Cixous et d'Eugénie Lemoine-Luccioni : la question féministe $y$ est, du fait même, pointée du doigt.

De Bellefeuille prend du métier (serait-il en train de devenir un excellent styliste?). D'où le contraste entre un propos lié à un questionnement de la modernité et un mouvement de phrase particulièrement bien équilibré, presque classique dans sa facture:

voici: je redoute la verge claire du père, celle qui "s'érige" plutôt, celle qui semble s'y plaire alors qu'aussitôt ça retraite, qu'aussitôt ça recule, hoquetant sa petite mort et, pour peu, s'y mouchant, par devoir, par prescription.

je voulais que tout ça soit enfin problématisé : du texte au totem, de l'anus à l'achat, de la jeune sœur à son désir. alors je lis qu'ailleurs le mot "totem" a pour sens "vulve de ma scur". la prescription est belle. elle a l'effet du meurtre. elle s'impose ici comme négation de la famille. [...]

HMH nous annonce depuis longtemps une vaste anthologie de la poésie québécoise du $\mathrm{XIX}^{\ominus}$ siècle. Le paradoxe n'est pas mince d'avoir été dépassé par les Franco-Américains. Paul P. Chassé publie une Anthologie de la poésie tranco-américaine de la Nouvelle-Angleterre (The Rhode Island Bicentennial Commission). Trois sections: $X I X^{e}$ siècle sous le titre des "Poètes de l'exil"; une section intitulée "Les Lauréats", réservée à des noms connus (Henri D'Arles, Louis Dantin et Rosaire Dion-Lévesque) et le $X X^{e}$ siècle. Le tout constituant un ensemble de près de 300 pages. Avis aux historiens de la littérature et aux bibliophiles.

Dans son introduction, l'auteur débat de la querelle entre régionalistes et universalistes, mais pour finir sur une note d'admiration (triste?) à l'endroit des poètes américains anglophones qui avaient su, il y a deux siècles, détourner leur regard du passé et foncer vers l'avant. Mais toute la problématique demeure. S'il est un idéal de devenir profondément américain, y lit-on, faut-il entendre le terme "au sens national ou continental »? Lorsque l'on est à cheval sur une frontière, ces questions doivent nécessairement demeurer insolubles.

Jean Fisette

UQAM 